\title{
Large transverse momentum dilepton production in heavy ion collisions with two-photon processes
}

\author{
Yong-Ping $\mathrm{Fu}^{1)}$, and Yun-De $\mathrm{Li}^{2}$ ) \\ Department of Physics, Yunnan University, Kunming 650091, China \\ 1) ynufyp@sina.cn; 2) yndxlyd@163.com
}

\begin{abstract}
The cold component of large transverse momentum dilepton production via semi-coherent twophoton interaction is calculated. The cold contribution is essential to the dilepton spectra in the soft region for different mass bins. The results are compared with the PHENIX experimental data at RHIC, and we find that the modification of semi-coherent two-photon processes is more evident with the rising dilepton mass bins.

PACS numbers:12.38.Mh, 25.75.Nq, 21.65.Qr
\end{abstract}

\section{INTRODUCTION}

The main propose of ultrarelativistic heavy ion collisions is to probe the thermal information of the dense thermalized matter which is named quark-gluon plasma (QGP).The electromagnetic radiation emitted from the center of the collision is a kind of very clean information due to the real and virtual photons which do not interact strongly, therefore photons and dileptons can escape from the dense medium without strong interactions, and may carry the information of the center dynamics[1-10]. However, so far no evident experiments show that some information is exactly produced from the thermalized medium [11 21].

The $\rho$ meson couples to the $\pi^{+} \pi^{-}$channel strongly, and the lifetime of $\rho$ meson is much shorter than the one of the expected hot hadronic gas. The yield of the $\rho$ mess spectrum may be modified in the hot medium due to the chiral symmetry restoration. The scenario of mass dropping or melting in a hot medium successfully interprets the dilepton yield enhancement in the low mass region at Super Proton Synchrotron (SPS) for Pb-Au $158 \mathrm{GeV} / \mathrm{A}$ collisions 22 28]. Recently, the measurement of the dilepton continuum at Relativistic Heavy Ion Collider (RHIC) energies has been performed by the PHENIX experiments for Au-Au $200 \mathrm{GeV} / \mathrm{A}$ collisions [29, 30]. The dilepton yield in the low mass range between 0.2 and $0.8 \mathrm{GeV}$ is enhanced by a factor of $2 \sim 3$ compared with the expectation from hadron decays. However, such a modifying scenario can not well explain the enhancement in the Au-Au collisions at RHIC. The imperfect modifying models of hadron decays present other probable mechanisms to explain the enhancement of the dilepton yield at low mass. Moreover, the dilepton enhancement at RHIC is implied that such phenomena are related strongly to the hot plasma scenario.

The contribution of thermal dileptons at the low mass is covered by the cocktail of hadron decays because the vector meson peaks is more pronounced than the thermal spectrum. The thermal information is dominant in the intermediate mass region between the $\phi$ and $J / \Psi$ vector meson for the phase transition theory, but the contribution of dileptons in this region also can be explained by the decays of charmed mesons. The NA60 collaboration has also observed an enhancement at intermediate mass. The data suggest that such an enhancement may include a thermal information and not just charm decays [31].

Except for the above problems, a new puzzle was performed by PHENIX collaboration [32]. The $P_{T}$ spectra of dileptons in $p+p$ collisions for different mass bins are in agreement with the expectation of the cocktail, charm decays and direct contributions. The agreement also exists in the Au-Au collisions at high $P_{T}\left(P_{T}>1 \mathrm{GeV}\right)$. The excess contribution above the cocktail and charm decays is consistent with the contribution from direct dileptons. In the soft region of $P_{T}<1 \mathrm{GeV}, \mathrm{pQCD}$ is out of use, and the spectra are still higher than the expectation of cocktail and charm decays. If fitted with exponential in this soft window, one has a slope $T_{\text {eff }} \approx 100 \mathrm{MeV}$, which is almost twice smaller than the typical slope of the standard thermal component. Another candidate in the soft window is the new "cold" component which is still valid in the low $P_{T}$ region. In Ref [33] the authors suggest a new cold dilepton production mechanism which is not discussed in the standard theory list before.

\section{GENERAL FORMALISM}

The semi-coherent two-photon processes are the so-called dilepton source for small mass bins and low $P_{T}$. Shuryak E. et. al. use the equivalent photon approximation to determine the differential cross section for the $\gamma \gamma$ processes in $\mathrm{Au}-\mathrm{Au}$ collisions, and have concluded that the semi-coherent production of dileptons does not contribute significantly to the PHENIX data[33]. However,we derive the yield of dileptons for $\gamma \gamma$ processes with the restriction $b \ll 2 R_{\perp}$, and finally find that the production of double photon interaction has a positive contribution to the cocktail and charm 
decays in the soft region. One notes that the impact parameter $b$ is smaller than the transverse dimension of the system $R_{\perp}$, and that the dense hot medium can not be created in the heavy ion collisions if $b>2 R_{\perp}$ because the QGP is centrality dependent.

The equivalent photon spectrum corresponding to a point charge $Z e$, moving with a velocity $v$ is given by

$$
n(\omega)=Z^{2} \frac{2 \alpha}{\pi v^{2}}\left[\xi K_{0} K_{1}-\frac{v^{2} \xi^{2}}{2}\left(K_{1}^{2}-K_{0}^{2}\right)\right],
$$

where the argument of the modified Bessel functions is $\xi=\omega R_{\min } / \gamma v$, and $R_{\min }$ corresponds to the radius of the radiation system with the maximum energy of the photons. After the MacDonald approximation the above formulation can be written into a compact form as 34,35

$$
n(\omega)=\frac{2 Z^{2} \alpha}{\pi} \ln \frac{\gamma}{\omega R_{\min }},
$$

where $R_{\text {min }} \sim 7 \mathrm{fm}$ and $\gamma=106$ for $\mathrm{Au}-\mathrm{Au} 200 \mathrm{GeV} / \mathrm{A}$ collisions at RHIC. In this article we use the natural units, namely $h=c=1$. The differential cross section of two-photon interaction for $\mathrm{Au}-\mathrm{Au}$ collisions is given by

$$
d \sigma=\sigma_{\gamma \gamma} d n_{1} d n_{2}
$$

where the total cross section of $\gamma \gamma \rightarrow e^{+} e^{-}$is 36 ]

$$
\sigma_{\gamma \gamma}=\frac{4 \pi \alpha^{2}}{M^{2}} \hat{\beta}_{L}\left[\frac{3-\hat{\beta}_{L}^{4}}{2 \hat{\beta}_{L}} \ln \frac{1+\hat{\beta}_{L}}{1-\hat{\beta}_{L}}-\left(2-\hat{\beta}_{L}^{2}\right)\right],
$$

and

$$
\hat{\beta}_{L}=\left[1-\frac{4 m_{e}^{2}}{M^{2}}\right]^{1 / 2}
$$

In the semi-coherent case, $q_{1 T} \gg q_{2 T}$, the total transverse momentum of dileptons is $\vec{P}_{T}=\vec{q}_{1 T}+\vec{q}_{2 T} \approx \vec{q}_{1 T}$, where $q_{i T}$ is the transverse momentum of a photon. Therefore the differential cross section can be written in the terms of dilepton transverse momentum as the following

$$
\begin{aligned}
\frac{d \sigma}{d^{2} p_{T} d M d y}= & 2 \pi\left(\frac{2 Z^{2} \alpha}{\pi}\right)^{2} \frac{1}{Z} \ln \frac{\gamma}{P_{T} R_{\min }} \frac{\sigma_{\gamma \gamma}}{p_{T}^{3}} \\
& \times \int_{p_{2 T \text { min }}}^{\gamma / R_{\min }} \ln \frac{\gamma}{q_{2 T} R_{\min }} \frac{1}{q_{2 T}} d q_{2 T}
\end{aligned}
$$

where the minimum transverse momentum of photon $q_{2}$ is $q_{2 \text { Tmin }} \sim 0.2 \mathrm{GeV}$ due to the single track acceptance condition. The factor $1 / Z$ is the charge form factor. The form factor for low momentum coherent photon is $F^{2}\left(q_{T} \rightarrow\right.$ $0) \sim 1$. However, for the incoherent photon, the form factor is $F^{2}\left(q_{T}>0\right) \approx \frac{1}{Z^{2}} Z[33]$. If both the photon transverse momenta $\left(q_{1 T}\right.$ and $\left.q_{2 T}\right)$ are the same large (non-coherent) or small (coherent), the total transverse momentum would have a very small value $\left(\left|\vec{P}_{T}\right|=\left|\vec{q}_{1 T}-\vec{q}_{2 T}\right| \rightarrow 0\right)$, then a dilepton could not obtain large transverse momentum in the $\gamma \gamma \rightarrow e^{+} e^{-}$interaction. The single track condition $q_{2 T} \geq 0.2 \mathrm{GeV}$ allows us to discuss large transverse momentum dilepton production in the two-photon reaction, the contribution of non-coherent and coherent photonphoton processes is weak compared with the semi-coherent processes. This is the reason why the semi-coherent approach is essential in the two-photon interactions.

In the semi-coherent case $\left(q_{1}=\left(\omega_{1}, q_{1 T}, q_{1 Z}\right)\right.$ and $\left.q_{2} \simeq\left(\omega_{2}, \overrightarrow{0},-q_{2 Z}\right)\right)$, a non-coherent photon with large transverse momentum is radiated from a nucleus and a coherent photon with small transverse momentum is radiated from a proton of another nucleus in the relativistic heavy ion collisions. The large transverse momentum yield for different mass bins is given as follows

$$
\frac{d N}{d^{2} p_{T} d y}=\int_{m_{1}}^{m_{2}} \frac{d N}{d^{2} p_{T} d M d y} d M
$$

where the yield relates to the differential cross section $d \sigma / d^{2} p_{T} d M d y$ for nucleus-nucleus collisions with the total cross section $\sigma_{t o t}$ in the form $d N / d^{2} p_{T} d M d y=\left(1 / \sigma_{t o t}\right)\left(d \sigma / d^{2} p_{T} d M d y\right)$. The authors in Ref[33] use $\sigma_{t o t} \sim 1.4 \times 10^{4} \mathrm{GeV}-2$ for the Au-Au $200 \mathrm{GeV} / \mathrm{A}$ collisions at RHIC. 


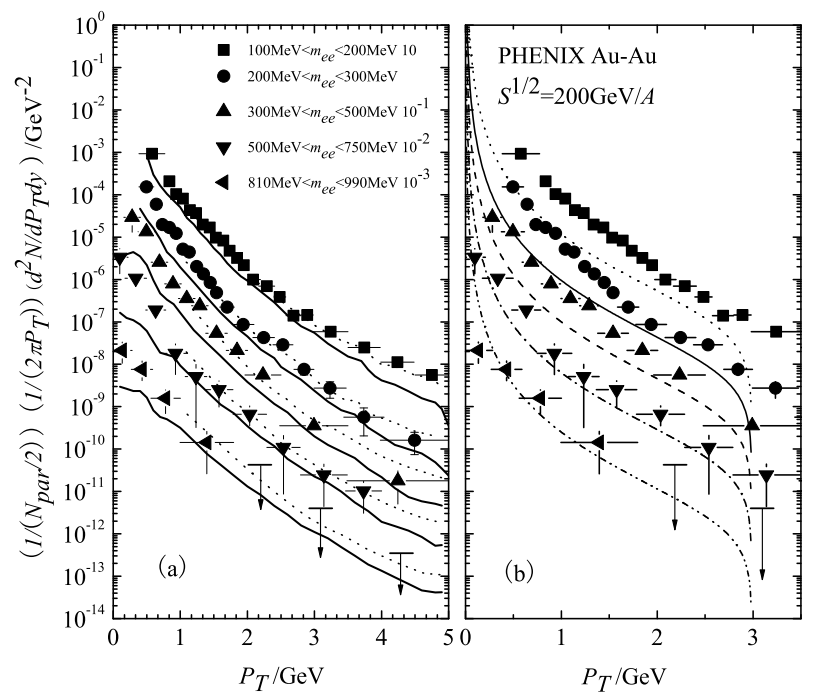

FIG. 1: The cold component dilepton production via the two-photon processes $\gamma \gamma \rightarrow e^{+} e^{-}$for different mass bins. (Panel a)The solid line: the sum of cocktail and charm decay; the dot line: the sum of cocktail and charm decay plus the contribution of direct dileptons [32]; (Panel b)the contribution of $\gamma \gamma \rightarrow e^{+} e^{-}$, the dot line: $100 \mathrm{MeV}<m_{e e}<200 \mathrm{MeV}$; the solid line: $200 \mathrm{MeV}<m_{e e}<300 \mathrm{MeV}$; the dash line: $300 \mathrm{MeV}<m_{e e}<500 \mathrm{MeV}$; the dash dot line: $500 \mathrm{MeV}<m_{e e}<750 \mathrm{MeV}$; the dash dot dot line: $810 \mathrm{MeV}<m_{e e}<990 \mathrm{MeV}$.

\section{NUMERICAL RESULTS}

From Fig. 1 one can see that the contribution of two-photon processes is only valid in the region of $P_{T}<3 \mathrm{GeV}$ due to the radiation limit condition $\omega<\gamma / R_{\min }$. It also implies that the spectra of $\gamma \gamma$ interaction depend on the nucleus radiation energies. The energy of photons radiated from nuclei is localized in the range of relatively small $q_{i T}$. Shuryak E. et. al. have discussed the two-photon processes by using a charge distribution form factor [33]. A charge distribution is necessary to the form factor, but the Woods-Saxon approximation they used has depressed the value of the form factor, therefore the spectra of dileptons under $\gamma \gamma \rightarrow e^{+} e^{-}$interaction are also depressed quickly with the rising dilepton transverse momentum. In this article we derive the yield of dileptons for $\gamma \gamma$ processes with the restriction $b \ll 2 R_{\perp}$, where the quark-gluon plasma may be created, and it is found that the spectra for different mass bins are essential in the soft region.

At low mass, $m_{e e}<200 \mathrm{MeV}$, the contribution of cocktail and charm decays is in agreement with the PHENIX data. However, in the soft region $\left(P_{T}<1 \mathrm{GeV}\right)$ the data are still higher than the expectation of cocktail and charm decays at $m_{e e}>200 \mathrm{MeV}$. The yield enhancement is more evident with the increasing of dilepton mass. In order to avoid the influence of $e^{+} e^{-}$decays of narrow resonance vector mesons, the mass regions around the $\omega$ meson and $\phi$ meson are excluded by PHENIX Collaboration.

In Fig. 2 we plot the large transverse momentum dilepton yield under $\gamma \gamma \rightarrow e^{+} e^{-}$interactions for different mass bins. As we discussed above the yield enhancement is not evident for the mass bins as $100 \mathrm{MeV}<m_{e e}<200 \mathrm{MeV}$ and $200 \mathrm{MeV}<m_{e e}<300 \mathrm{MeV}$, so the correlation of cold $\gamma \gamma$ component is weak; In the mass region of $300 \mathrm{MeV}<$ $m_{e e}<500 \mathrm{MeV}, 500 \mathrm{MeV}<m_{e e}<750 \mathrm{MeV}$ and $810 \mathrm{MeV}<m_{e e}<990 \mathrm{MeV}$ the data are higher almost one order than the expectation of cocktail and charm decays, and the modification of $\gamma \gamma$ reaction is essential now. Therefore the two-photon interaction plays an important role in the large transverse momentum dilepton production in the soft $P_{T}$ region.

\section{CONCLUSION}

We derived the two-photon processes from the passage of $\gamma \gamma \rightarrow e^{+} e^{-}$. The large transverse momentum dilepton can be created in the semi-coherent two-photon interaction. The $\gamma \gamma$ processes may be an essential complementarity to the previous standard cold dilepton production. In the MacDonald approximation the energy of the radiated photon is limited in the region of $\omega<\gamma / R_{\text {min }}$, therefore the cold dilepton component of two-photon processes is still valid in the soft $P_{T}$ region where the pQCD is out of use. 


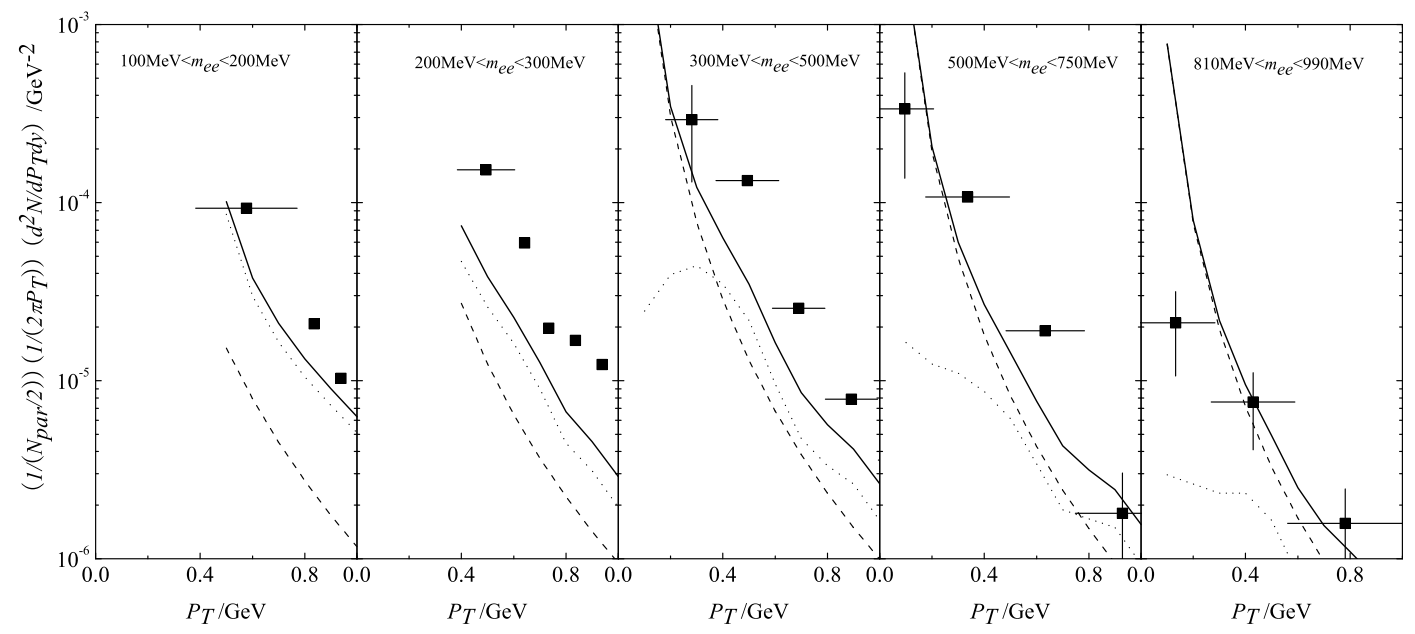

FIG. 2: The dilepton spectra of two-photon processes for different mass bins. The dilepton transverse momentum is in the soft region $P_{T}<1 \mathrm{GeV}$. The dot line is the contribution of the cocktail and charm decay, the dash line is the photon-photon production, and the solid line is the total contribution.

The numerical results are plotted by comparing with the PHENIX experimental data. The modification of twophoton processes is more evident with the rising dilepton mass, especially in the mass bins of $300 \mathrm{MeV}<m_{e e}<500 \mathrm{MeV}$, $500 \mathrm{MeV}<m_{e e}<750 \mathrm{MeV}$ and $810 \mathrm{MeV}<m_{e e}<990 \mathrm{MeV}$.

This work is supported by the National Natural Science Foundation of China (10665003 and 11065010).

[1] Kajantie K, Kapusta J, Mclerran L et al. Phys. Rev. D, 1986, 34: 2746

[2] Ruuskanen P V. Nucl. Phys. A, 1992, 544: 169

[3] Rapp R, Shuryak E. Phys. Lett. B, 2000, 473: 13

[4] Shuryak E, Xiong L. Phys. Rev. Lett., 1993, 70: 2241

[5] Hung C M, Shuryak E. Phys. Rev. C, 1998, 57: 1891

[6] Shuryak E V, Zahed I. Phys. Rev. C, 2004, 70: 021901

[7] Lévai P, Müller B, Wang X N. Phys. Rev. C, 1995, 51: 3326

[8] Li G Q, Gale C. Phys. Rev. Lett., 1998, 81: 1572

[9] Wong C Y. Phys. Rev. D, 1984, 30: 961

[10] Bjorken J D. Phys. Rev. D, 1983, 27: 140

[11] Alam J, Srivastava D K, Sinha B et al. Phys. Rev. D, 1993, 48: 1117

[12] Fries R J, Müller B, Srivastava D K. Phys. Rev. Lett., 2003, 90: 132301

[13] Kapusta J, Lichard P, Seibert D. Nucl. Phys. A, 1992, 544: 485

[14] Bass S A, Müller B, Srivastava D K. Phys. Rev. Lett., 2003, 90: 082301

[15] Srivastava D K, Sinha B. Phys. Rev. Lett., 1994, 73: 2421

[16] Chatterjee R, Frodermann E S, Heinz U et al. Phys. Rev. Lett., 2006, 96: 202302

[17] Jeon S, Marian J J, Sarcevic I. Nucl. Phys. A, 2003, 715: 795

[18] Kolb P F, Sollfrank J, Heinz U. Phys. Rev. C, 2000, 62: 054909

[19] Adler S S et al (PHENIX Collaboration). Phys. Rev. Lett., 2005, 94: 232301; Adler S S et al (PHENIX Collaboration). Phys. Rev. Lett., 2003, 91: 072301

[20] Albrecht R et al (WA80 Collaboration). Phys. Rev. Lett., 1996, 76: 3506

[21] Büsching $\mathrm{H}$ et al (PHENIX Collaboration). Nucl. Phys. A, 2006, 774: 103

[22] Hung C M, Shuryak E V. Phys. Rev. C, 1997, 56: 453

[23] Kaempfer B, Koch P, Pavlenko O P. Phys. Rev. C, 1994, 49: 1132

[24] Song C. Phys. Rev. C, 1993, 47: 2861

[25] Srivastava D K, Sinha B. Phys. Rev. Lett., 1994, 73: 2421

[26] Gale C, Kapusta J I. Nucl. Phys. B, 1991, 357: 65

[27] Gale C, Lichard P. Phys. Rev. D, 1994, 49: 3338

[28] Srivastava D K, Sinha R, Gale C. Phys. Rev. C, 1996, 53: 567

[29] Toia A. Eur. Phys. J. C, 2007, 49: 243; Toia A et al (PHENIX Collaboration). Nucl. Phys. A, 2006, 774: 743

[30] Shahoian R. PoS, 2006, HEP2005: 131 
[31] Arnaldi R et al (NA60 Collaboration). arXiv:0806.0577 [nucl-ex]

[32] Adare A et al (PHENIX Collaboration). Phys. Rev. C, 2010, 81: 034911

[33] Staig P, Shuryak E. arXiv:1005.3531 [nucl-th]

[34] Jackson J D. Classical Electrodynamics. New York: John Wiley, 1975

[35] Baur G, Hencken K, Trautmann D. J. Phys. G, 1998, 24: 1657

[36] Manuel D, Godbole R M, Nowakowski M et al. Phys. Rev. D, 1994, 50: 2335 